\title{
Risk factors and prognostic index model for pancreatic cancer
}

\author{
Hui Huang ${ }^{1}$, Jichun Sun ${ }^{1}$, Zheming Jiang ${ }^{2}$, Xianlin Zhang ${ }^{3}$, Zheng Li $^{3}$, Hongwei Zhu ${ }^{1}$, Xiao Yu ${ }^{1 \wedge}$ \\ ${ }^{1}$ Department of Hepatopancreatobiliary Surgery, The Third Xiangya Hospital, Central South University, Changsha, China; ${ }^{2}$ Department of \\ Ultrasound, Hunan Provincial Maternal and Child Health Care Hospital Changsha, China; ${ }^{3}$ Department of General Surgery, Affiliated Renhe \\ Hospital of China, Three Gorges University, Yichang, China \\ Contributions: (I) Conception and design: H Huang, H Zhu, X Yu; (II) Administrative support: None; (III) Provision of study materials or patients: \\ None; (IV) Collection and assembly of data: H Huang, Z Jiang, X Zhang, Z Li; (V) Data analysis and interpretation: H Huang, H Zhu, J Sun, Z \\ Jiang, X Zhang, Z Li; (VI) Manuscript writing: All authors; (VII) Final approval of manuscript: All authors. \\ Correspondence to: Xiao Yu; Hongwei Zhu. Department of Hepatopancreatobiliary Surgery, The Third Xiangya Hospital, Central South University, \\ Changsha 410013, China. Email: xiaoyuxybio@163.com; zhuhongweizhw0509@126.com.
}

Background Pancreatic cancer is a highly malignant tumor with poor prognosis. Chronic inflammation contributes to the progression of pancreatic cancer. However, few studies have examined the prognostic role of inflammatory markers in this cancer. Our study sought to analyze the prognostic risk factors of and construct a prognostic index (PI) model using inflammatory markers for pancreatic cancer.

Methods: Forty-eight patients diagnosed with pancreatic cancer at our hospital were selected for this retrospective analysis. Data on the general clinical characteristics, tumor-related features, blood index factors, and treatment methods were collected. The Cox proportional-hazards model was used to analyze the factors affecting the prognosis, and the Kaplan-Meier analysis was used to draw the survival curve.

Results: The median overall survival time was 14.5 months, and the 1-, 2-, and 3-year survival rates were $20.83 \%$ (10/48), 6.25\% (3/48), and 4.17\% (2/48), respectively. The univariate analysis showed that tumor grade, vascular invasion, adjacent tissue invasion, lymph node metastasis, tumor-node-metastasis (TNM) stage, the neutrophil-lymphocyte ratio (NLR), the platelet-lymphocyte ratio (PLR), and the lymphocytemonocyte ratio (LMR) were significantly correlated with the median survival of pancreatic cancer patients $(\mathrm{P}<0.05)$. The Cox regression equation showed that tumor grade III-IV (X1), vascular invasion (X2), TNM stage III-IV (X3), a NLR >3.8 (X4), and a PLR >182.1 (X5) were independent risk factors affecting the prognosis of patients with pancreatic cancer (all $\mathrm{P}<0.05$ ). The prognostic model for pancreatic cancer can be expressed as: $\mathrm{PI}=3.521 \mathrm{X} 1+4.157 \mathrm{X} 2+1.282 \mathrm{X} 3+2.441 \mathrm{X} 4+6.015 \mathrm{X} 5$. Patients with tumor grade I-II, nonvascular invasion, TNM stage I-II, a NLR $\leq 3.8$, and a PLR $\leq 182.1$ exhibited a higher 1 -year survival rate. The areas under the receiver operating characteristic (ROC) curves for the NLR $>3.8$ and the PLR $>182.1$ were 0.778 and 0.713 , respectively.

Conclusions: Tumor grade, vascular invasion, TNM staging, the NLR, and the PLR are independent risk factors affecting the prognosis of pancreatic cancer patients. The NLR and PLR have good clinical value in predicting the survival outcomes of pancreatic cancer patients.

Keywords: Pancreatic cancer; prognosis; risk factors; prognostic index model (PI model)

Submitted Nov 22, 2021. Accepted for publication Jan 11, 2022.

doi: $10.21037 /$ gs-21-848

View this article at: https://dx.doi.org/10.21037/gs-21-848

^ ORCID: 0000-0001-6557-2382. 


\section{Introduction}

In recent years, with the continuous improvement of people's living standards, the prevalence of pancreatic cancer has gradually increased worldwide, and it has become the second most common cause of death from gastrointestinal-related tumors (1). Pancreatic cancer, which is a highly malignant tumor of the digestive system, has no obvious symptoms at the beginning of the disease; thus, early diagnosis rate is low. Most patients are already at an advanced stage when diagnosed, which leads to treatment difficulties and a poor prognosis (2). Surgical resection is still the main treatment for pancreatic cancer, but the resection rate is only $10-20 \%(3,4)$. Additionally, due to the complexity of the pancreatic anatomy, the operation is risky and difficult, and the postoperative mortality of patients is as high as $5-10 \%$ (5). The question of how to improve the survival rate of patients with pancreatic cancer has posed a difficult problem for a long time. Thus, it is of great significance to explore the risk factors affecting the prognosis of patients with pancreatic cancer.

Prognostic markers assist to stratify patients who would benefit from surgery and formulate therapeutic strategies for patients with tumors (6). Previous studies have confirmed that pancreatic cancer tumor size, the location, the degree of invasion, the degree of differentiation, and the pathological stage have important clinical significance in predicting recurrence, metastasis, and prognosis (7). However, these well-established prognostic parameters are highly dependent on histological examinations and are only available for assessment after surgery. In addition, $\mathrm{Ma}$ et al. constructed a risk model based on 6 KRAS-associated metabolic genes to predict survival of pancreatic cancer with high accuracy (8). Other prognostic index (PI) models using multi-omics integrated analysis of microRNA or DNA methylation markers were also established $(9,10)$. Whereas, detection and analysis of the genetic factors is difficult to widely applied in clinical practice. Therefore, a more accurate and accessible risk index system needs to be established to predict the prognosis of patients with pancreatic cancer.

Relevant information shows that about $20 \%$ of malignant tumors are closely related to non-controllable inflammation, and chronic inflammation is involved in the occurrence and development of cancer (11). Various systemic inflammatory markers are potent prognostic factors for cancer and are readily to obtain in routine clinical practice. Several studies have reported that systemic immune inflammatory markers, such as the neutrophil-to-lymphocyte ratio (NLR), plateletlymphocyte ratio (PLR), and lymphocyte-to-monocyte ratio
(LMR) have important roles in the prediction of pancreatic cancer survival $(12,13)$. However, available evidence does not support PLR as a reliable prognostic factor for overall survival in patients with resectable pancreatic cancer (14). Besides, Chawla et al. reported that pretherapy NLR and PLR do not predict survival in patients who underwent pancreatectomy for pancreatic cancer (15). Therefore, no consensus has been achieved regarding their prognostic roles in pancreatic cancer partially due to the different in sample size, parameter cutoff value, treatment modality or genetic heterogeneity in these studies (13). This study retrospectively analyzed the clinical data of pancreatic cancer patients to identify the combined effect of clinicopathological factors and systemic inflammatory markers (including NLR, PLR and LMR) on the prognosis of patients, and established an index model to predict the survival of patients and further to analyze the predictive efficiency of the screened risk factors on 1-year survival of the patients. We present the following article in accordance with the STARD reporting checklist (available at https://gs.amegroups.com/article/ view/10.21037/gs-21-848/rc).

\section{Methods}

\section{Patient characteristics}

Forty-eight eligible patients diagnosed with pancreatic cancer at the Third Xiangya Hospital from January 2016 to July 2018 were retrospectively enrolled in this study (see Figure 1).

To be eligible for inclusion in this study, patients had to meet the following inclusion criteria: (I) have undergone radical resection for pancreatic cancer, and have postoperative histopathology confirmed pancreatic cancer; (II) have not received any antibiotic anti-inflammatory therapy, radiotherapy, chemotherapy, or other treatments before surgery; and (III) have complete clinical data and follow-up data available. Patients were excluded from the study if they met any of the following exclusion criteria: (I) had other malignant tumors or distant metastases; (II) had hepatic and renal dysfunction; (III) died perioperatively; (IV) had autoimmune diseases or infectious diseases; and/or (V) had recently used glucocorticoid or other immunosuppressive drugs. All procedures performed in this study involving human participants were in accordance with the Declaration of Helsinki (as revised in 2013). The study was approved by Ethics Committee of The Third Xiangya Hospital [No. SYXK(Xiang)2017-0002]. Individual consent for this retrospective analysis was waived. 


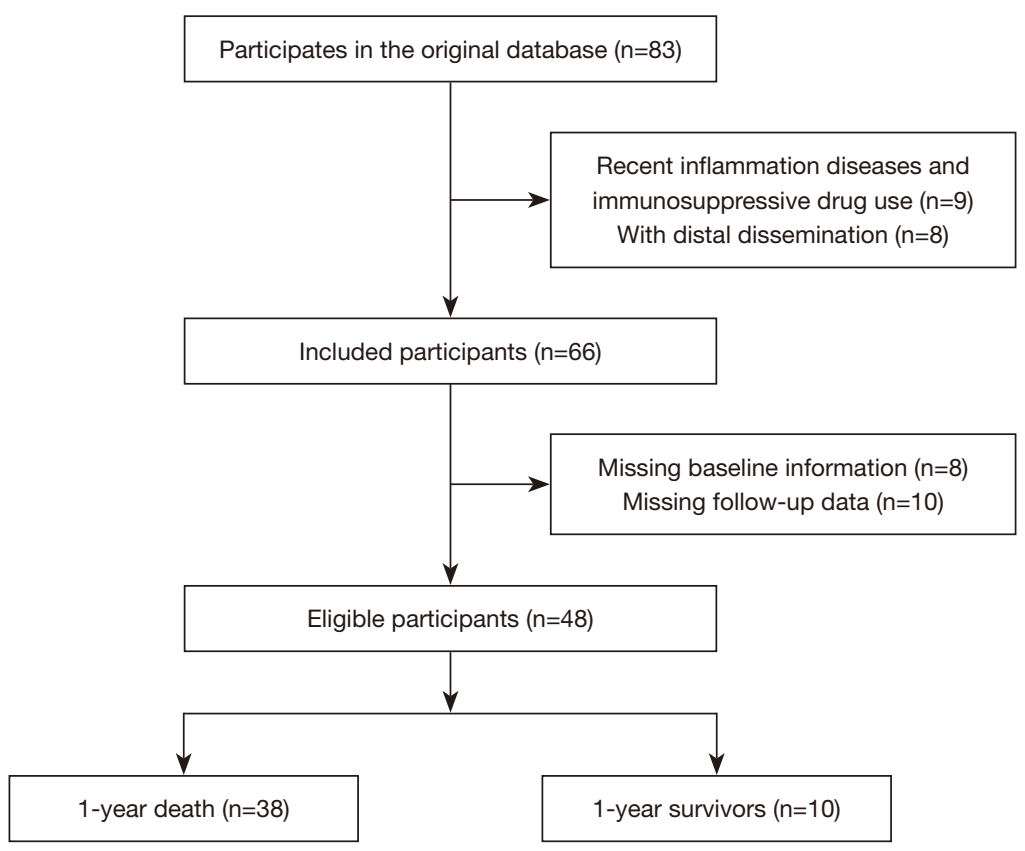

Figure 1 Flow diagram of the inclusion criteria for patients.

\section{Data collection}

Data on general clinical factors, tumor-related factors, systemic inflammatory index factors, and treatment methods were retrospectively collected from patient information system when patients were admitted to our hospital. The general clinical factors included age, gender, body mass index (BMI), smoking history, drinking history, diabetes, and hypertension. The tumor-related factors included tumor size, tumor location, tumor grade, tumor-node-metastasis (TNM) stage, and whether there was vascular invasion, adjacent tissue invasion, and lymph node metastasis. The systemic inflammation index included NLR, PLR, and LMR, which were calculated based on the ratio of absolute counts of neutrophils, lymphocytes, platelet and monocytes, respectively. After admission, all patients underwent radical resection, including pancreaticoduodenectomy, distal pancreatectomy, and total pancreatectomy. TNM staging was based on the $7^{\text {th }}$ edition criteria developed by the American Joint Council on Cancer.

\section{Follow-up}

All patients received standard follow-up, and they were followed up in 2 ways: outpatient or telephone appointments. The follow-up was performed every 3 months for the first
2 years after surgery, and every 3-6 months thereafter until December 2021 or death. The 1-, 2-, and 3-year survival rate and the median survival time were recorded, and the factors affecting the postoperative survival rate were analyzed.

\section{Statistical analysis}

The data were analyzed using IBM SPSS20.0 software. The general data and other measurement data of the 2 groups are represented as mean \pm standard deviation, and the independent sample $t$-test was used. For mean comparisons between the 2 groups, the count data are all expressed as number (percentage), and the $\chi^{2}$ test was used to compare the data between the 2 groups. The significant variables $(\mathrm{P}<0.05)$ in univariate analysis further entered the multivariate Cox proportional-hazards regression model, and the significant variables in Cox model were identified as prognostic risk factors affecting the prognosis of the patients. Further, the PI model was constructed by the screened prognostic risk factors with the odds ratio [Exp (B)] as coefficient of these parameters. Kaplan-Meier method was used to draw the survival curves, and the comparisons of the survival rates were performed using the log-rank test. The receiver operating characteristic (ROC) curve was used to evaluate the predictive value of the prognostic factors on the survival outcome, and the cutoff values of the NLR, 
Table 1 General information of the patients

\begin{tabular}{|c|c|}
\hline General information & Values \\
\hline Age (years) & $57.26 \pm 12.31$ \\
\hline \multicolumn{2}{|l|}{ Gender } \\
\hline Male & $23(47.92)$ \\
\hline Female & 25 (52.08) \\
\hline BMI $\left(\mathrm{kg} / \mathrm{m}^{2}\right)$ & $22.03 \pm 3.26$ \\
\hline \multicolumn{2}{|l|}{ Smoking history } \\
\hline Yes & $20(41.67)$ \\
\hline No & $28(58.33)$ \\
\hline \multicolumn{2}{|l|}{ Drinking history } \\
\hline Yes & $22(45.83)$ \\
\hline No & $26(54.17)$ \\
\hline \multicolumn{2}{|l|}{ Diabetes } \\
\hline Yes & 19 (39.58) \\
\hline No & $29(60.42)$ \\
\hline \multicolumn{2}{|l|}{ Hypertension } \\
\hline Yes & $11(22.92)$ \\
\hline No & $37(77.08)$ \\
\hline Tumor size (mm) & $43.62 \pm 26.84$ \\
\hline \multicolumn{2}{|l|}{ Tumor site } \\
\hline Pancreatic head & $32(66.67)$ \\
\hline Pancreatic body and tail & $16(33.33)$ \\
\hline \multicolumn{2}{|l|}{ Tumor grade } \\
\hline Grade I-II & $22(45.83)$ \\
\hline Grade III-IV & $26(54.17)$ \\
\hline \multicolumn{2}{|l|}{ Vascular invasion } \\
\hline Yes & $23(47.92)$ \\
\hline No & $25(52.08)$ \\
\hline \multicolumn{2}{|l|}{ Adjacent tissue invasion } \\
\hline Yes & 27 (56.25) \\
\hline No & $21(43.75)$ \\
\hline \multicolumn{2}{|l|}{ Lymph node metastasis } \\
\hline Yes & $24(50.00)$ \\
\hline No & $24(50.00)$ \\
\hline \multicolumn{2}{|l|}{ TNM staging } \\
\hline Stage I-II & $16(33.33)$ \\
\hline Stage III-IV & 32 (66.67) \\
\hline
\end{tabular}

Data are represented as mean \pm SD or $n(\%)$.
PLR, and LMR in predicting the 1-year prognosis were calculated based on Youden's index (the maximum value of specificity + sensitivity -1 ) of the ROC analysis. A P value $<0.05$ was considered statistically significant.

\section{Results}

\section{Baseline characteristics}

The median survival time of the 48 patients was 14.5 months, and the 1-, 2-, and 3-year survival rates were $20.83 \%(10 / 48)$, $6.25 \%$ (3/48), and $4.17 \%$ (2/48), respectively. Among the 48 patients, $47.92 \%$ were male and $52.08 \%$ were female, and the patients had an average age of $57.26 \pm 12.31$ years. For other general information, please see Table 1 .

\section{Univariate analysis of the risk factors affecting prognosis}

The univariate analysis showed that tumor grade, vascular invasion, adjacent tissue invasion, lymph node metastasis, TNM staging, the NLR, the PLR, and the LMR were significantly related to the median survival of patients with pancreatic cancer $(\mathrm{P}<0.05$; Table 2$)$.

\section{Multivariate analysis of the risk factors affecting the prognosis of pancreatic cancer}

The risk factors of statistical significance in the univariate analysis were put into the Cox regression equation. It was concluded that tumor grade III-IV (X1), vascular invasion (X2), TNM stage III-IV (X3), a NLR >3.8 (X4), and a PLR $>182.1$ (X5) were independent risk factors affecting the prognosis of pancreatic cancer patients $(\mathrm{P}<0.05)$ (Table 3). The fitting expression of the prognostic risk function model for pancreatic cancer patients was the PI $=3.521 \mathrm{X} 1+4.157 \mathrm{X} 2$ $+1.282 \mathrm{X} 3+2.441 \mathrm{X} 4+6.015 \mathrm{X} 5$.

\section{Survival analysis}

As Figure 2 shows, the 1-year survival rate of patients with tumor grades I-II was significantly higher than that of patients with tumor grades III-IV; the 1-year survival rate of patients without vascular invasion was significantly higher than that of patients with vascular invasion; the 1-year survival rate of patients with TNM stage I-II was significantly higher than that of patients with stage III-IV; the 1 -year survival rate of patients with a NLR $\leq 3.8$ was significantly higher than that of patients with a NLR $>3.8$; 
Table 2 Univariate analysis of the risk factors affecting prognosis

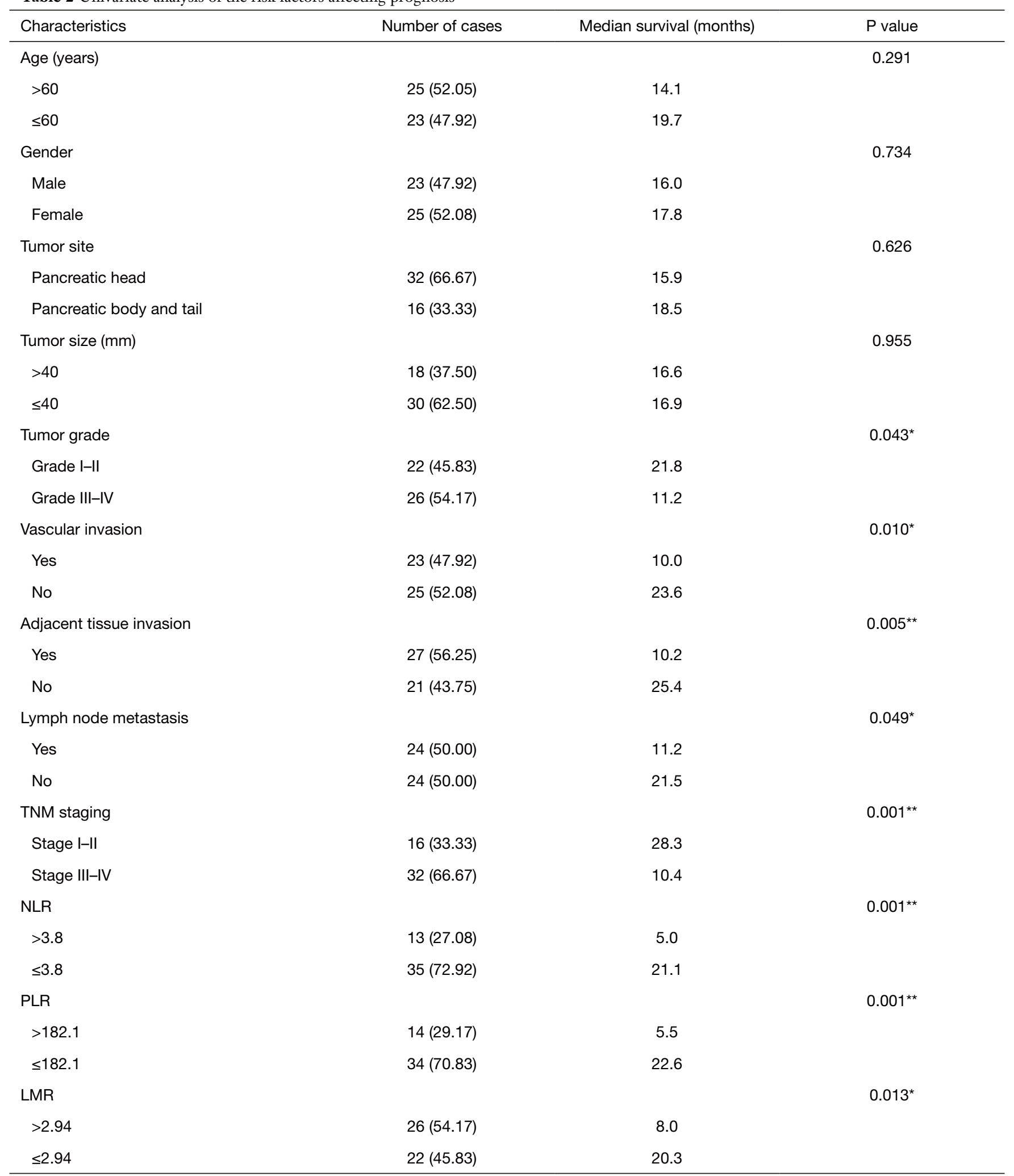

\footnotetext{
*, $\mathrm{P}<0.05$ indicates a significant difference; ${ }^{*}, \mathrm{P}<0.01$ indicates an extremely significant difference. NLR, neutrophil-lymphocyte ratio; PLR,
} platelet-lymphocyte ratio; LMR, lymphocyte-monocyte ratio. 
Table 3 Multivariate analysis of the risk factors affecting the prognosis of pancreatic cancer

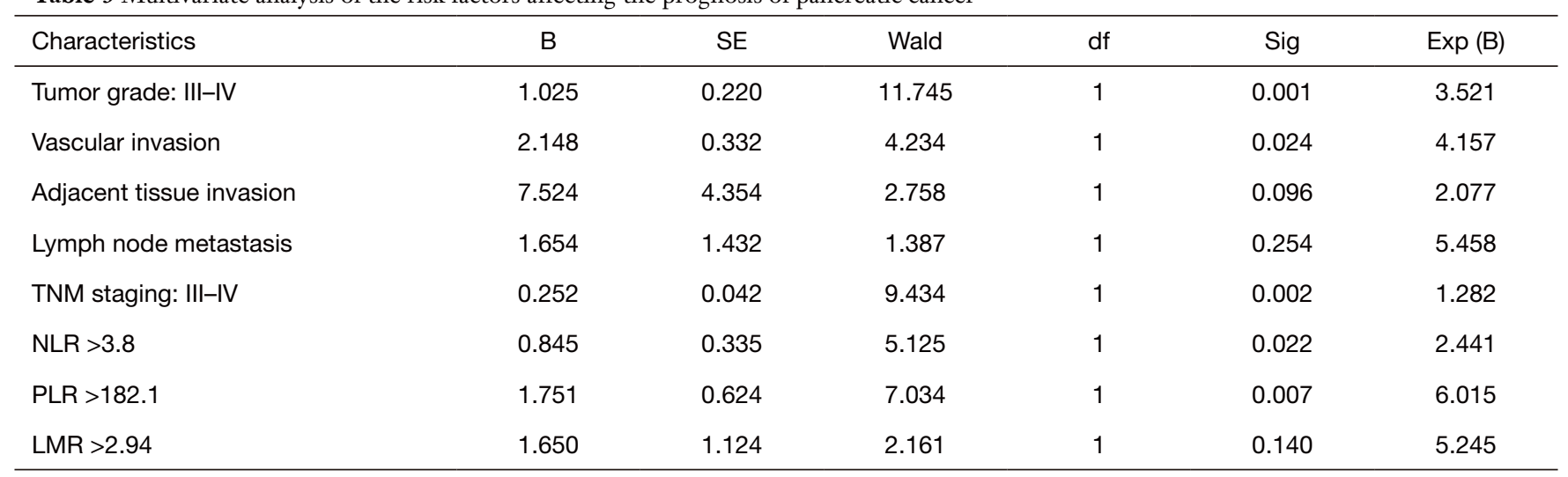

NLR, neutrophil-lymphocyte ratio; PLR, platelet-lymphocyte ratio; LMR, lymphocyte-monocyte ratio.

and the 1-year survival rate of patients with a PLR $\leq 182.1$ was significantly higher than that of patients with a PLR $>182.1$. The differences between the factors are shown in Table 4.

\section{ROC curves assess the predictive value of the prognostic factors on the survival outcomes}

The ROC curve analysis showed that the prognostic factors of a NLR $>3.8$ and a PLR $>182.1$ had good value in predicting the survival outcomes of pancreatic cancer patients (Table 5 and Figure 3).

\section{Discussion}

Pancreatic cancer, which is a common digestive system tumor, has the characteristics of an insidious onset, rapid progression, a low resection rate, and a poor prognosis. Research has shown that the survival rate of patients with pancreatic cancer decreases over time (16). The American Cancer Center reported that the 5-year survival rate of patients with pancreatic cancer was $<6 \%$, and the median survival time was only 6 months (17). It is of great significance to understand the clinical characteristics of pancreatic cancer and analyze the factors affecting the prognosis of patients to prolong the survival period. Our study revealed that tumor grade, vascular invasion, TNM stage, NLR >3.8 and a PLR >182.1 were independent risk factors affecting the prognosis of pancreatic cancer patients.

It is commonly believed that histological parameters and invasion status are important risk factors affecting the prognosis of pancreatic cancer. Dr. El Nakeeb and other scholars have found that a tumor diameter $>2 \mathrm{~cm}$ is an independent risk factor for the prognosis of pancreatic cancer (18). In addition, some scholars believe that lymph node metastasis is an independent risk factor for the postoperative survival of pancreatic cancer patients, and the 5 -year survival rate of pancreatic cancer patients with lymph node metastasis has been shown to be significantly lower than that of patients without lymph node metastasis (19). This study did not find that tumor size and lymph node metastasis were factors affecting the prognosis of patients with pancreatic cancer, which may be related to the number of collected cases and lymph node dissection. For a long time, tumor histological grade has been used by different scholars as an indicator to evaluate the prognosis of pancreatic cancer. Studies have confirmed that pancreatic cancer with high grades or poorly differentiated pancreatic cancer have a higher recurrence and mortality rate than pancreatic cancer with poorly differentiated grades (20). In this study, pancreatic cancer tumor grades III-IV were found to be risk factors affecting patient prognosis, which confirms the importance of tumor histological grading in the prognostic evaluation of pancreatic cancer. Most studies have reported that patients with pancreatic cancer with vascular invasion have a poor prognosis, but a few scholars believe that the prognosis of patients was not associated with vascular invasion (21). Research shows that the tumor invasion of blood vessels causes tumor cells to form micrometastases, and new blood vessels can provide nutrition for tumor growth, thus forming a vicious cycle (22). Due to the special anatomical location of pancreatic cancer, patients tend to wait until the onset of abdominal pain, jaundice, and other symptoms before seeking a diagnosis, 

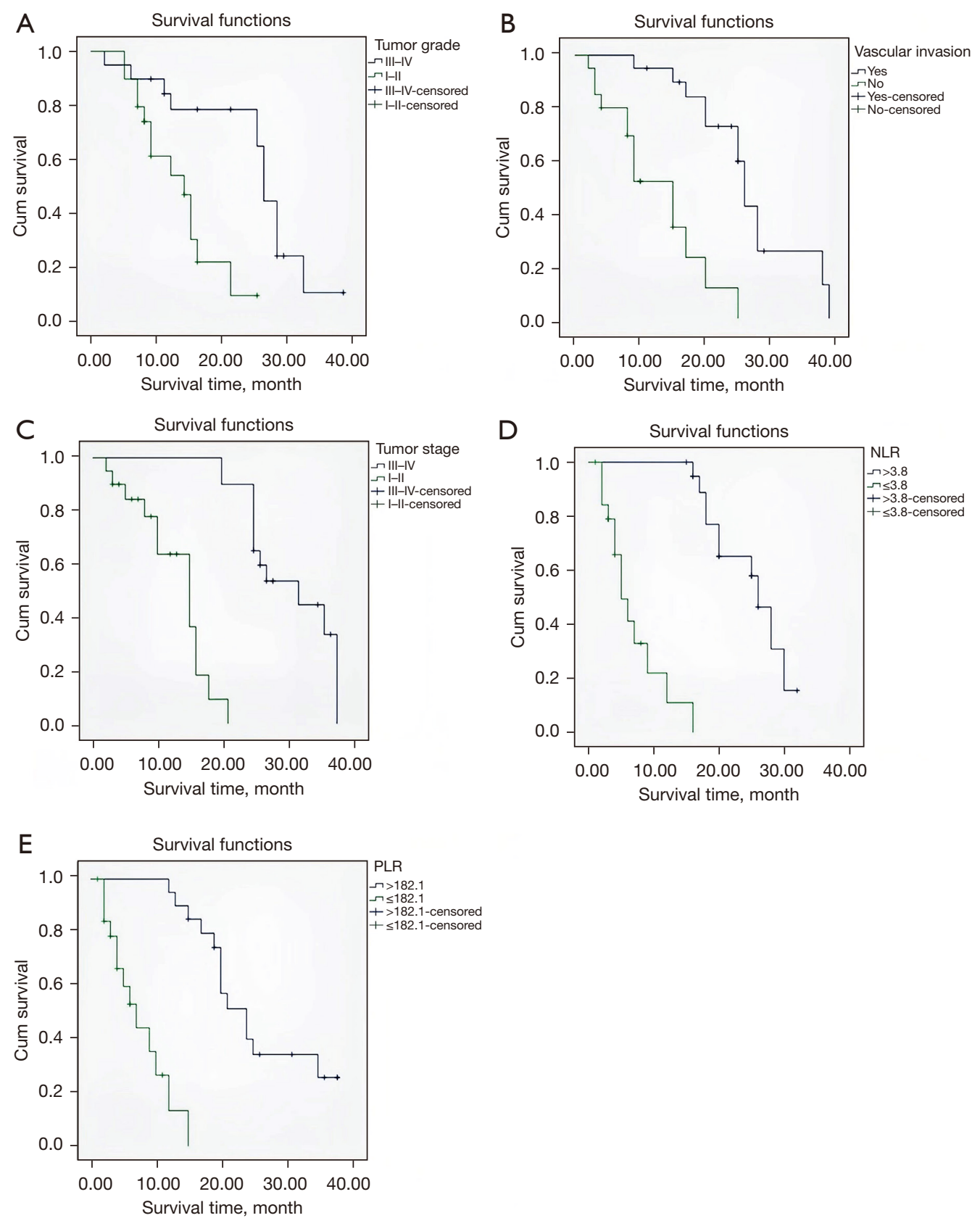

Figure 2 Survival rate analysis. (A) Survival curves of patients with different tumor grades; (B) survival curves of patients with or without vascular invasion; (C) survival curves of patients with different tumor stages; (D) survival curves of patients with different NLR; (E) survival curves of patients with different PLR. NLR, neutrophil-lymphocyte ratio; PLR, platelet-lymphocyte ratio.

which means that the best opportunity for treatment is lost. TNM staging can be used to evaluate the resectability of the tumor before surgery. Patients with higher staging have a worse prognosis, which can inform clinicians' choice of an appropriate treatment plan, and thus prolong the survival time of patients (23).

In recent years, studies have confirmed that systemic inflammation is a critical factor affecting the occurrence and development of all tumors, which inducing cancer proliferation and metastasis or promoting angiogenesis 
Table 4 Differences between various factors

\begin{tabular}{lccc}
\hline Characteristics & $\mathrm{P}$ & $\mathrm{HR}$ & \multicolumn{1}{c}{$\mathrm{P5 \%} \mathrm{Cl}$} \\
\hline Tumor grade: I-II vs. III-IV & $<0.001$ & 3.412 & $2.241-4.875$ \\
Vascular invasion: No vs. Yes & $<0.001$ & 2.524 & $1.102-5.527$ \\
TNM staging: I-II vs. III-IV & $<0.001$ & 4.214 & $2.021-7.874$ \\
NLR $\leq 3.8$ vs. NLR $>3.8$ & $<0.001$ & 1.521 & $1.136-3.547$ \\
PLR $\leq 182.1$ vs. PLR $>182.1$ & $<0.001$ & 3.432 & $1.120-5.796$ \\
\hline
\end{tabular}

NLR, neutrophil-lymphocyte ratio; PLR, platelet-lymphocyte ratio.

Table 5 ROC curves to evaluate the predictive value of the prognostic factors on survival outcomes.

\begin{tabular}{lllllcc}
\hline Characteristics & AUC & SE & P & $95 \% \mathrm{Cl}$ & Sensibility & Specificity \\
\hline Tumor grade: III-IV & 0.605 & 0.037 & 0.010 & $0.525-0.671$ & 58.90 & 46.72 \\
Vascular invasion & 0.631 & 0.045 & 0.009 & $0.527-0.672$ & 64.30 & 50.80 \\
TNM staging: III-IV & 0.687 & 0.039 & $<0.001$ & $0.583-0.725$ & 67.92 & 49.20 \\
NLR $>3.8$ & 0.778 & 0.032 & $<0.001$ & $0.640-0.778$ & 91.11 & 75.80 \\
PLR $>182.1$ & 0.713 & 0.037 & $<0.001$ & $0.585-0.726$ & 75.02 & 57.55 \\
\hline
\end{tabular}

ROC, receiver operating characteristic; NLR, neutrophil-lymphocyte ratio; PLR, platelet-lymphocyte ratio.

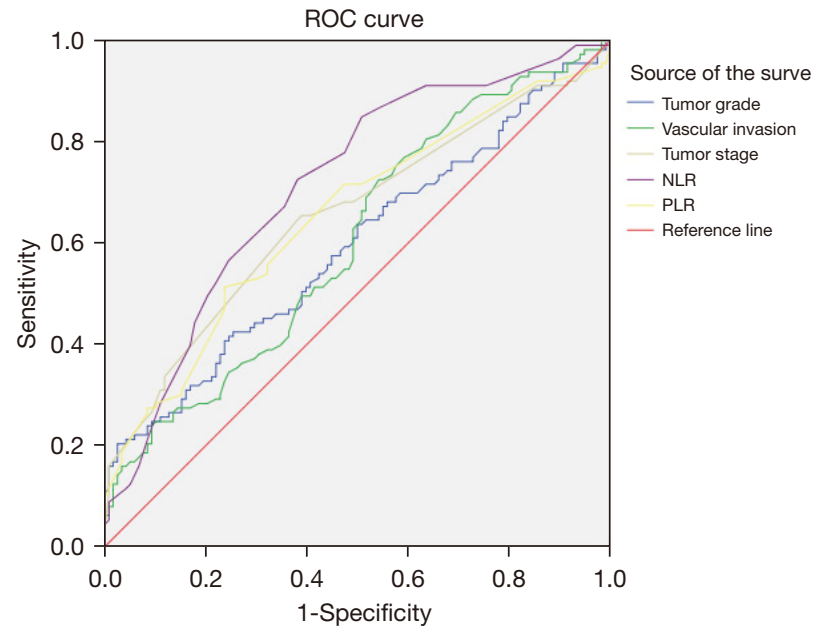

Figure 3 ROC curve to evaluate the predictive value of the prognostic factors on the survival outcome. ROC, receiver operating characteristic; NLR, neutrophil-lymphocyte ratio; PLR, platelet-lymphocyte ratio.

and repairing DNA damage $(13,24)$. NLR and PLR are important inflammatory indicators. In patients with colon cancer, prostate cancer, lung cancer, and other malignant tumors, an increase in the levels of these 2 indicators are considered a sign of poor prognosis, which can reflect the pro-tumor inflammatory response and balance between anti-tumor immune status. Neutrophils can regulate the activity of natural killer cells and lymphocytes (25). An increase in their number usually indicates the proliferation of tumor cells, while a decrease in the number of lymphocytes often indicates abnormal host immune function and weakened anti-tumor immunity. Lymphopenia provides a necessary environment for tumor invasion and metastasis. Most patients with malignant tumors have elevated platelets. Platelets spread tumor cells to other parts of the body by releasing thrombospondin and other factors, which can lead to the occurrence of immune reactions and an increased PLR (26). The NLR and PLR have been shown to be related to the prognosis of a variety of solid malignancies (27). Turker et al. reported that high NLR is independent adverse prognostic factors for overall survival in metastatic pancreatic cancer (25). Basal high NLR $>3$, rather than PLR, was an independent poor prognostic factor in metastatic pancreatic cancer (28). Moreover, NLR is a potentially independent prognostic factors for overall survival in resectable pancreatic cancer patients (29). While, Chawla et al. concluded that pretherapy neutrophil to lymphocyte ratio and platelet to lymphocyte ratio do not predict survival in resectable pancreatic cancer (15). Our study revealed that NLR and PLR were prognostic 
factors affecting the survival in resectable pancreatic cancer. However, this study is limited to the retrospective nature and small sample size, and further internal and external validations are needed to test the prediction efficiency of the current PI in pancreatic cancer using similar inclusion and exclusion criteria.

In conclusion, tumor grade, vascular invasion, TNM stage, the NLR, and the PLR are independent risk factors affecting the prognosis of patients with pancreatic cancer, and the NLR and the PLR have good clinical value in predicting the survival outcomes of patients with pancreatic cancer.

\section{Acknowledgments}

Funding: This research received funding from the National Natural Science Foundation of China (No. 81873589) and the National Natural Science Foundation of China (No. 82000614).

\section{Footnote}

Reporting Checklist: The authors have completed the STARD reporting checklist. Available at https://gs.amegroups.com/ article/view/10.21037/gs-21-848/rc

Data Sharing Statement: Available at https://gs.amegroups. com/article/view/10.21037/gs-21-848/dss

Conflicts of Interest: All authors have completed the ICMJE uniform disclosure form (available at https://gs.amegroups. com/article/view/10.21037/gs-21-848/coif). The authors have no conflicts of interest to declare.

Ethical Statement: The authors are accountable for all aspects of the work in ensuring that questions related to the accuracy or integrity of any part of the work are appropriately investigated and resolved. All procedures performed in this study involving human participants were in accordance with the Declaration of Helsinki (as revised in 2013). The study was approved by Ethics Committee of The Third Xiangya Hospital [No. SYXK(Xiang)2017-0002]. Individual consent for this retrospective analysis was waived.

Open Access Statement: This is an Open Access article distributed in accordance with the Creative Commons Attribution-NonCommercial-NoDerivs 4.0 International License (CC BY-NC-ND 4.0), which permits the non- commercial replication and distribution of the article with the strict proviso that no changes or edits are made and the original work is properly cited (including links to both the formal publication through the relevant DOI and the license). See: https://creativecommons.org/licenses/by-nc-nd/4.0/.

\section{References}

1. Hsueh HY, Pita-Grisanti V, Gumpper-Fedus K, et al. A review of physical activity in pancreatic ductal adenocarcinoma: Epidemiology, intervention, animal models, and clinical trials. Pancreatology 2022;22:98-111.

2. Moore A, Donahue T. Pancreatic Cancer. JAMA 2019;322:1426.

3. Nipp RD, Zanconato A, Zheng H, et al. Predictors of Early Mortality After Surgical Resection of Pancreatic Adenocarcinoma in the Era of Neoadjuvant Treatment. Pancreas 2017;46:183-9.

4. Stathis A, Moore MJ. Advanced pancreatic carcinoma: current treatment and future challenges. Nat Rev Clin Oncol 2010;7:163-72.

5. Chen J, Wang S, Jia S, et al. Integrated Analysis of Long Non-Coding RNA and mRNA Expression Profile in Pancreatic Cancer Derived Exosomes Treated Dendritic Cells by Microarray Analysis. J Cancer 2018;9:21-31.

6. Kubo H, Murakami T, Matsuyama R, et al. Prognostic Impact of the Neutrophil-to-Lymphocyte Ratio in Borderline Resectable Pancreatic Ductal Adenocarcinoma Treated with Neoadjuvant Chemoradiotherapy Followed by Surgical Resection. World J Surg 2019;43:3153-60.

7. Zhou B, Xu JW, Cheng YG, et al. Early detection of pancreatic cancer: Where are we now and where are we going? Int J Cancer 2017;141:231-41.

8. Ma Z, Li Z, Ma Z, et al. Development of a KRASAssociated Metabolic Risk Model for Prognostic Prediction in Pancreatic Cancer. Biomed Res Int 2021;2021:9949272.

9. Jia Y, Shen M, Zhou Y, et al. Development of a 12-biomarkers-based prognostic model for pancreatic cancer using multi-omics integrated analysis. Acta Biochim Pol 2020;67:501-8.

10. Kong L, Liu P, Fei X, et al. A Prognostic Prediction Model Developed Based on Four CpG Sites and Weighted Correlation Network Analysis Identified DNAJB1 as a Novel Biomarker for Pancreatic Cancer. Front Oncol 2020;10:1716.

11. Murata M. Inflammation and cancer. Environ Health Prev Med 2018;23:50. 
12. Kawai M, Hirono S, Okada KI, et al. Low lymphocyte monocyte ratio after neoadjuvant therapy predicts poor survival after pancreatectomy in patients with borderline resectable pancreatic cancer. Surgery 2019;165:1151-60.

13. Yang JJ, Hu ZG, Shi WX, et al. Prognostic significance of neutrophil to lymphocyte ratio in pancreatic cancer: a meta-analysis. World J Gastroenterol 2015;21:2807-15.

14. Riauka R, Ignatavicius P, Barauskas G. Preoperative Platelet to Lymphocyte Ratio as a Prognostic Factor for Resectable Pancreatic Cancer: A Systematic Review and Meta-Analysis. Dig Surg 2020;37:447-55.

15. Chawla A, Huang TL, Ibrahim AM, et al. Pretherapy neutrophil to lymphocyte ratio and platelet to lymphocyte ratio do not predict survival in resectable pancreatic cancer. HPB (Oxford) 2018;20:398-404.

16. Qadan M, Fernandez-Del Castillo C. Can Prognosis Be Modified in Pancreatic Cancer? Ann Surg Oncol 2020;27:632-3.

17. Bartell N, Bittner K, Vetter MS, et al. Role of Endoscopic Ultrasound in Detecting Pancreatic Cancer Missed on Cross-Sectional Imaging in Patients Presenting with Pancreatitis: A Retrospective Review. Dig Dis Sci 2019;64:3623-9.

18. El Nakeeb A, El Shobary M, El Dosoky M, et al. Prognostic factors affecting survival after pancreaticoduodenectomy for pancreatic adenocarcinoma (single center experience). Hepatogastroenterology 2014;61:1426-38.

19. Bramhall SR, Allum WH, Jones AG, et al. Treatment and survival in 13,560 patients with pancreatic cancer, and incidence of the disease, in the West Midlands: an epidemiological study. Br J Surg 1995;82:111-5.

20. Licata A, Montalto G, Soresi M. Pancreatic cancer: risk and preventive factors. Intern Emerg Med 2018;13:321-3.

21. Torres SM, Vaz da Silva DG, Ribeiro HSC, et al. Shortterm outcomes after vascular resection for pancreatic

Cite this article as: Huang $\mathrm{H}$, Sun J, Jiang Z, Zhang X, Li Z, Zhu H, Yu X. Risk factors and prognostic index model for pancreatic cancer. Gland Surg 2022;11(1):186-195. doi: 10.21037/ gs-21-848 tumors: Lessons learned from 72 cases from a single Brazilian Cancer Center. J Surg Oncol 2020;121:857-62.

22. Viallard C, Larrivée B. Tumor angiogenesis and vascular normalization: alternative therapeutic targets. Angiogenesis 2017;20:409-26.

23. Kawai M, Fukuda A, Yogo A, et al. A case of a malignant serous neoplasm of the pancreas with synchronous vascular invasion and metachronous metastases. Clin J Gastroenterol 2020;13:1347-54.

24. Kumarasamy C, Sabarimurugan S, Madurantakam RM, et al. Prognostic significance of blood inflammatory biomarkers NLR, PLR, and LMR in cancer-A protocol for systematic review and meta-analysis. Medicine (Baltimore) 2019;98:e14834.

25. Turker S, Cilbir E, Guven DC, et al. The relation between inflammation-based parameters and survival in metastatic pancreatic cancer. J Cancer Res Ther 2021;17:510-5.

26. Schwartz PB, Poultsides G, Roggin K, et al. PLR and NLR Are Poor Predictors of Survival Outcomes in Sarcomas: A New Perspective From the USSC. J Surg Res 2020;251:228-38.

27. Sit D, Raissi T, Giuliani M, et al. Use of the neutrophil to lymphocyte ratio (NLR) and the platelet to lymphocyte ratio (PLR) for prognostication of patients with earlystage non-small cell lung cancer (NSCLC) treated with stereotactic body radiation therapy (SBRT): a systematic review. J Radiat Oncol 2019;8:13-21.

28. Dogan M, Algin E, Guven ZT, et al. Neutrophillymphocyte ratio, platelet-lymphocyte ratio, neutrophilplatelet score and prognostic nutritional index: do they have prognostic significance in metastatic pancreas cancer? Curr Med Res Opin 2018;34:857-63.

29. Cheng H, Luo G, Lu Y, et al. The combination of systemic inflammation-based marker NLR and circulating regulatory $T$ cells predicts the prognosis of resectable pancreatic cancer patients. Pancreatology 2016;16:1080-4. 